\title{
Tatiana KASPERSKI, Les politiques de la radioactivité : Tchernobyl et la mémoire nationale en Biélorussie contemporaine. Paris : Éditions Pétra, 2020, $346 \mathrm{p}$.
}

\author{
Eva DERONT \\ Docteure en science politique \\ Chercheure associée \\ UMR Pacte \\ Université Grenoble Alpes (FR) \\ eva.deront@umrpacte.fr
}

Doi : 10.5077/journals/connexe.2021.e600

L'ouvrage Les Politiques de la radioactivité : Tchernobyl et la mémoire nationale en Biélorussie contemporaine est une adaptation de la thèse de doctorat de Tatiana Kasperski. À travers ce travail, l'auteure cherche à comprendre les mécanismes de construction - mais surtout d'effacement - de la mémoire de la catastrophe de Tchernobyl, alors que la Biélorussie relance depuis quelques années l'utilisation de la technologie nucléaire pour la production d'électricité.

Pour ce faire, l'auteure a organisé son propos de manière principalement chronologique. L'ouvrage se compose ainsi de cinq parties. La première détaille le manque de préparation et de transparence des autorités soviétiques lors de la catastrophe, qui, pour dissimuler leurs failles, vont construire un récit fondé surl'héroïsation etl'abnégation des populations de l'URSS. La deuxième partie analyse l'évolution de la mémoire de Tchernobyl lors de l'accès à l'indépendance, suite à la dislocation de l'URSS, moment où émergent de nouveaux acteurs et de nouveaux répertoires d'action. La troisième partie met l'accent sur l'instrumentalisation de la mémoire de Tchernobyl pour la construction d'un sentiment national biélorusse, que ce soit par le nouveau pouvoir d'Alexandre Loukachenko (élu en 1994) ou par ses opposants, de plus en plus réprimés. L'analyse entamée se prolonge dans la quatrième partie, à travers le développement d'exemples de formes de manifestations de la mémoire sous Loukachenko. Enfin, la dernière partie met en exergue la dépolitisation de la catastrophe par le choix d'instruments de politiques publiques particuliers, ainsi que par la mise en œuvre de politiques de responsabilisation des individus.

L'ensemble de l'ouvrage appuie la thèse selon laquelle la mémoire de Tchernobyl est subordonnée à la construction identitaire d'une nation biélorusse, menée de façon autoritaire par l'État (d'abord sous l'URSS puis en tant que nation indépendante), avec peu d’influence de la part d'autres acteurs politiques. Pour l'auteure, la construction du sens autour de la catastrophe de Tchernobyl a été largement influencée par la nécessité de définir les contours de l'unité nationale biélorusse. Entre 1986 et 1989, les récits officiels de Tchernobyl sont marqués par le fatalisme ainsi que par l'héroïsation du dévouement de la population soviétique ; ces récits permettent ainsi aux autorités d'éviter l'analyse des responsabilités politiques dans le déroulement de la catastrophe. Cette dernière est 
présentée comme une bataille à mener contre un ennemi extérieur, hors de contrôle, sans rapport avec les défaillances de la société dans laquelle il surgit. Le peuple soviétique apparaît, lui, comme une "famille ", dont il faudrait isoler les comportements et les individus déviants et menaçant l'unité nationale. À ce moment, le projet identitaire «biélorusse » est porté par l'opposition politique nationaliste et prône l'indépendance vis-à-vis de l'URSS ; ce contre-récit présente la catastrophe comme un des crimes du régime soviétique, contre lequel il s'agit de construire un sentiment d'appartenance à « une communauté imaginaire » biélorusse.

La transformation du système politique au début des années 1990 permet l'émergence de nouveaux acteurs de protestation : des réseaux d'intellectuels (au sein des instances officielles) et des groupes informels (notamment de jeunes) portent des revendications concernant les problèmes environnementaux du pays ainsi que le patrimoine culturel biélorusse. Ces acteurs vont rejoindre ou soutenir la naissance du Front Populaire Biélorusse (FPB), qui s'est saisi de la question de la contamination radioactive des territoires dès 1989, à travers des cérémonies commémoratives, des meetings, des séminaires, des conférences, actions s'ajoutant à la contre-expertise des intellectuels et aux lettres de plainte des habitants.

Si le FPB parvient également à s'allier avec des associations caritatives chargées de l'aide aux victimes de la catastrophe (ce qui accroît sa visibilité et sa légitimité), il échoue à récupérer les mouvements locaux de protestation face à Tchernobyl : les mobilisations ouvrières du début des années 1990 (grèves ayant pour objectif le paiement de compensations ainsi que l'approvisionnement en produits alimentaires non contaminés) restent largement indépendantes du FPB et préfèrent orienter leurs demandes vers l'URSS. On peut regretter que l'auteure n'explique pas les raisons de ce décalage entre les interprétations nationalistes et les revendications locales des populations directement touchées ; elle souligne simplement une « distance géographique et sociale qui sépare les intellectuels de la capitale [...] et les populations locales » (p. 141). Une comparaison avec d'autres mobilisations aurait pu permettre de voir dans quelle mesure cette situation est spécifique à la mémoire de Tchernobyl et de mettre en exergue les ressorts expliquant ces faibles liens entre les mobilisations locales et celles chapeautées, nationalement, par le FPB.

L'auteure cherche par ailleurs à montrer la montée en généralité et l'accentuation des cadrages nationaux à travers les discours des acteurs visant à " nommer, blâmer et réclamer ». L'opposition réutilise ainsi la notion de «victime» de la catastrophe et pointe les responsabilités des autorités locales et soviétiques. Les nationalistes sont les seuls à prendre en charge des cérémonies commémoratives, qu’ils utilisent comme terreau pour nourrir le sentiment d'unité nationale; selon l'auteure, "la singularité de l'événement est progressivement minimisée puisque celui-ci est assimilé à d'autres événements tragiques de l'histoire nationale » (p. 147). L'auteure souligne également que l'inscription progressive des exigences des victimes dans un discours nationaliste permet une convergence des revendications et leur montée en généralité (p. 138) (demande de lois de protection sociale avec un calendrier précis de mise en œuvre plutôt que de mesures ponctuelles). Cette partie descriptive n'explique toutefois pas les raisons de cette montée en généralité, ni ne démontre de manière diachronique un glissement 
vers un discours nationaliste de la part des différents types d'acteurs étudiés.

Suite à l'élection de Loukachenko, cette période de « pluralisation de la mémoire » se termine : les manifestations sont interdites par les autorités et violemment réprimées par les forces de police, les forces politiques n'ont plus accès au parlement, lieu d'expression des revendications liées aux conséquences de l'accident. Le récit officiel de la catastrophe est alors construit et instrumentalisé pour légitimer le pouvoir. Pour les partisans comme pour les opposants de Loukachenko, la mémoire de la catastrophe de Tchernobyl se fait sous l'angle de la nation.

Loukachenko mobilise une interprétation positive de la catastrophe et des échecs des politiques mises en œuvre jusqu'alors, cherchant à « insuffler une vie nouvelle » aux territoires contaminés pour mettre fin aux politiques de dépeuplement, à diminuer le budget consacré à la gestion des conséquences de l'accident et à relancer la production agricole. Dans des cérémonies au fort culte de la personnalité, il incite les habitants à ne plus subir passivement les conséquences de la catastrophe, à se " prendre en main ». Les réfractaires sont traités comme des ennemis de l'État, qui refusent d'adhérer à l'unité nationale, décrite comme mise en péril par la contamination radioactive et les risques sanitaires. L'auteure souligne ainsi qu’à partir de 1995, les interprétations dominantes de l'accident sont passées d'un fort sentiment antisoviétique à la revalorisation du passé, témoignant de la volonté de Loukachenko de préserver des liens avec la Russie.

Les contestataires tentent, eux, de former une communauté nationale biélorusse autour du caractère violent et tragique du passé, renforçant ainsi la dimension antisoviétique de la mémoire nationaliste : des parallèles sont tissés entre Tchernobyl et les massacres de leaders biélorusses dans la ville de Kourapaty, sous Staline, ou encore entre Tchernobyl et la Grande Guerre Patriotique (la guerre de l'URSS contre l'Allemagne nazie). Selon l'auteure, la mémoire de Tchernobyl se trouve ainsi diluée dans d'autres événements fondant les récits identitaires biélorusses.

Autour de 2001, l'auteure note une forte diminution dans la participation aux manifestations organisées par l'opposition, suite à l'augmentation de la répression, au choix fait par les opposants d'investir leurs faibles ressources dans la campagne présidentielle ou dans des événements avec peu de participants. Au fil du temps, l'aspect mémoriel est relégué en second plan: Tchernobyl devient alors un instrument de contestation politique plus globale.

Dans un dernier chapitre thématique qui tranche avec l'ensemble plus chronologique, l'auteure cherche à étudier les particularités de la mémoire publique à travers le mode de gestion des conséquences de la catastrophe de Tchernobyl. Elle y analyse la dépolitisation de la mémoire de la catastrophe par la mise en œuvre de politiques de responsabilisation des individus : des dispositifs de zonage, l'enseignement de la radioprotection, ainsi que la responsabilisation des habitants amenés à gérer annuellement leur «budget de contamination » interne concourent à rendre "opaque le caractère politique et économique des décisions [...] et des enjeux liés à la pollution radioactive » (p. 243). L'auteure rejoint ainsi les critiques émises notamment par la sociologue Sezin Topçu : l'introduction d'une gouvernance néolibérale des risques sanitaires dans les zones contaminées, fondée sur des actions individuelles et sur le travail des individus sur 
eux-mêmes, renforce la déresponsabilisation de l'État dans la gestion de la catastrophe. Lélection de Loukachenko marque ainsi un changement de cap dans la politique de gestion des conséquences de Tchernobyl, avec la promotion du retour à la vie normale dans les territoires contaminés (qui va de pair avec une diminution des indemnisations octroyées aux victimes).

Cette démonstration permet à l'auteure de mettre en avant un « schisme mémoriel entre les mythes glorieux officiels, qui ont fini par dominer l'espace public, et les mémoires privées, qui n'ont pas de cadre pour être exprimées publiquement et encore moins pour servir de fondement à des revendications politiques » (p. 280). Ce schisme serait expliqué par les trois mécanismes suivants : la fermeture du régime politique, la promotion de politiques identitaires (qui minimisent les problèmes spécifiques des territoires les plus touchés) et la gestion de la contamination radioactive.

Même si l'on peut regretter que l’impact des structures d'opportunités politiques sur les usages de la mémoire ne soit pas clairement détaillé, faute d'indicateurs permettant d'analyser l'évolution du discours mémoriel, la lecture de cet ouvrage demeure riche pour quiconque s'intéresse à la gestion des catastrophes industrielles, alors que l'on commémore cette année les dix ans de la catastrophe de Fukushima. Les références à l'unité nationale, l'extériorisation de la menace et les politiques de responsabilisation des individus qui ont été mobilisées dans l'après Tchernobyl résonneront également de manière frappante avec la gestion actuelle de la crise sanitaire du coronavirus.

Open Access Publications - Bibliothèque de l'Université de Genève

Creative Commons Licence 4.0

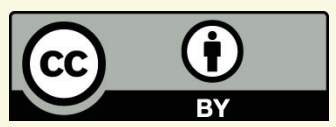

